\title{
Deforestación y fragmentación en las áreas más biodiversas de la Cordillera Occidental de Antioquia (Colombia)
}

\author{
Deforestation and fragmentation in the most biodiverse areas \\ in the Western Cordillera of Antioquia (Colombia)
}

\author{
Jennifer Calderón-Caro ${ }_{\square}^{\square}$, Ana María Benavides 주
}

\section{Resumen}

La degradación de los ecosistemas en los Andes, una de las regiones con mayor biodiversidad del mundo, es atribuida principalmente a la deforestación y la fragmentación. La implementación de áreas protegidas (AP) es una estrategia de conservación efectiva para mitigar los efectos de la transformación del paisaje sobre la biodiversidad. En la Cordillera Occidental de Colombia, en Antioquia, se estableció recientemente el Corredor del Oso de Anteojos (COA, 4169 km²), como una estrategia para la conservación de la biodiversidad. En este estudio, a partir de información derivada de sensores remotos y bases de datos, localizamos las áreas con mayor biodiversidad en este corredor, y analizamos si estás áreas están siendo protegidas. Los resultados indican que la deforestación $\left(162.37 \mathrm{~km}^{2}\right)$ y la fragmentación (> $90 \%$ ) en el COA durante los últimos 19 años se concentran en las áreas más biodiversas, al interior y por fuera de las AP. Si bien las AP alcanzan 30\% del área total del COA, las áreas de mayor biodiversidad están representadas en menos de un $17 \%$. Estos resultados indican el riesgo de pérdida de biodiversidad y la creciente necesidad de fortalecer las AP y delimitar nuevas áreas biodiversas.

Palabras clave. Fragmentación. Heterogeneidad climática. Índices Dinámicos de hábitat. Pérdida de bosque. Vulnerabilidad.

\begin{abstract}
The degradation of ecosystems in the Andes, one of the most biodiverse regions in the world, is caused by deforestation and forest fragmentation. The implementation of protected areas (PA) is an effective conservation strategy to mitigate the effects of landscape transformation on biodiversity. In the western mountain range of Colombia, in Antioquia, the Spectacled Bear Corridor (COA, $\left.\sim 4169 \mathrm{~km}^{2}\right)$ was recently established. We describe the state of forest conservation in the COA and analyze whether areas of high biodiversity are being protected or not, using information obtained from remote sensors and large databases. The results indicate that deforestation $\left(162.37 \mathrm{~km}^{2}\right)$ and fragmentation $(>90 \%)$ in the COA during the last 19 years are concentrated in the areas of greatest biodiversity, and occur both inside and outsidePA. Although protected areas reach $30 \%$ of the total area of the COA, the areas with the greatest biodiversity represent less than $17 \%$. These results indicate the risk of biodiversity loss and the growing need to strengthen PA and to delimit new biodiverse areas.
\end{abstract}

Keywords. Climatic heterogeneity. Dynamic Habitat Indices. Forest loss. Fragmentation. Vulnerability 


\section{Introducción}

La pérdida de biodiversidad se ha incrementado globalmente durante los últimos 20 años, debido, principalmente, a las presiones antropogénicas, como la deforestación, la fragmentación, los incendios, la sobreexplotación de recursos y el cambio climático, entre otras (Laurance et al., 2012). Este fenómeno ha llevado, a su vez, a la disminución de servicios ecosistémicos indispensables para el sostenimiento y el bienestar de la humanidad (Cardinale et al., 2012). En los Andes tropicales, la región con mayor biodiversidad del mundo (Myers et al., 2000) y que provee servicios ecosistémicos fundamentales para la supervivencia de cerca del $40 \%$ de la población de Suramérica (Banco Mundial, 2013), la degradación de sus ecosistemas naturales es atribuida a dos causas principales: (1) la deforestación ( 2.6 millones ha. de bosque perdidas anualmente; FAO, 2020) y (2) la fragmentación de los remanentes de bosque (Armenteras \& Villareal, 2002; Zhai et al. 2015; Barlow et al., 2016).

Una de las estrategias globales de conservación de la diversidad biológica más efectivas para mitigar los efectos de la transformación del paisaje es la implementación de áreas protegidas (AP) (Brooks et al., 2006; Venter et al., 2018). Actualmente, más de 700 millones de hectáreas de bosque a nivel global se encuentran en AP legalmente establecidas (FAO, 2020). En los últimos 15 años, los mecanismos globales de conservación exigen la identificación, el reconocimiento y la protección de nuevos sitios prioritarios o de importancia para la conservación de la biodiversidad (CBD, 2004; Jackson \& Kennedy, 2009). Actualmente, la delimitación de AP requiere incorporar las medidas de importancia biológica (e.g., número de especies endémicas, riqueza de especies, diversidad beta (Myers et al., 2000; Radeloff et al ., 2019) y medidas de vulnerabilidad del hábitat (e.g., pérdida de hábitat, deforestación y fragmentación; Hansen et al., 2013).

Adicionalmente a las medidas de importancia biológica y de vulnerabilidad, en los análisis de planificación de las AP en regiones con amplia heterogeneidad ambiental como los Andes tropicales (Kattan et al., 2004; Ramirez-Villegas et al., 2014), es importante incluir medidas que permitan conocer el cambio en la composición de especies a lo largo de gradientes ambientales de la región (i.e., diversidad beta o complementariedad). No obstante, en regiones tan poco muestreadas como los trópicos, medidas de heterogeneidad climática son útiles para reconocer el recambio de especies, al asumir que dos unidades del paisaje climáticamente diferentes tendrán un conjunto de especies disímiles, particularmente en montañas tropicales, donde los cambios en condiciones climáticas generan un marcado gradiente de diversidad (Ferrier, 2002; Soria-Auza \& Kessler, 2008; Slik et al., 2015; Gardner et al., 2020).

Si bien las medidas antes mencionadas (importancia biológica y vulnerabilidad) han sido utilizadas para la delimitación de áreas prioritarias para la conservación de la biodiversidad (Myers et al., 2000; Bruner et al., 2001; Ferrier, 2002; Orme et al., 2005; Brooks et al., 2006), aún hay AP que se encuentran ubicadas en lugares poco representativos de la biodiversidad local (Armenteras \& Villareal, 2002; Andam et al., 2008; Forero-Medina \& Joppa, 2010; Venter et al., 2018) o tienen un fuerte impacto por la transformación del paisaje (Armenteras \& Villareal, 2002; Leisher et al., 2013). En ese sentido, evaluar el estado de conservación y la representación de las áreas más biodiversas en las AP es indispensable para la gestión y la toma de decisiones que lleven a reducir la pérdida de la biodiversidad, bajo el escenario actual de cambio climático y la fuerte transformación del paisaje. En este estudio describimos el estado de conservación, en términos de vulnerabilidad (i.e. deforestación y fragmentación de los bosques), en el Corredor del Oso de Anteojos (Tremarctos ornatus) en el suroeste y occidente de Antioquia, Colombia, y analizamos la representatividad de las áreas de alta importancia biológica en las AP. El Corredor del Oso de Anteojos (COA), ubicado en una de las regiones mejor preservadas de Antioquia, nació como una estrategia social, que ha sido promovida a nivel gubernamental por medio de una ordenanza departamental para la conservación de la fauna silvestre y la flora de la región desde el año 2017.

\section{Materiales y métodos}

\section{Área de estudio}

El COA delimita $\sim 4169.7 \mathrm{~km}^{2}$ en el suroeste y occidente del departamento de Antioquia, en la Cordillera Occidental de Colombia (Figura S1a). Está ubicado en una de las regiones con mayor extensión de bosques y, aunque ha sido poco estudiado, se considera una de las regiones con mayor diversidad biológica en Antioquia (González-Caro \& Vásquez, 2017; Triviño et al., 2018). El COA presenta una precipitación media anual de $\sim 2400$ mm.año $0^{-1}$ y una temperatura media anual de $\sim 15^{\circ} \mathrm{C}$ (Karger et al., 2017). El corredor abarca principalmente ecosistemas de bosques andinos y altoandinos, en un rango de elevación entre 2600 y 3900 msnm. En el 40\% 
del área del COA $\left(\sim 1676.57 \mathrm{~km}^{2}\right)$ se encuentran seis figuras de protección, que corresponden a trece AP: tres Distritos Regionales de Manejo Integrado (DRMI), un Parque Nacional Natural (PNN), un Parque Natural Regional (PNR), tres Reservas Forestales Protectoras Nacionales (RFPN) y cinco Reservas Naturales de la Sociedad Civil (RNSC) (Figura S1a, Tabla S1). Las figuras de protección de estas AP difieren principalmente en la permisibilidad para la ejecución de actividades económicas dentro de su jurisdicción (Decreto 2372 del 2010, Ministerio de Medio Ambiente y Desarrollo Territorial de Colombia). Los límites de las AP en formato vector fueron obtenidos de los datos abiertos de Parques Nacionales Naturales de Colombia.

\section{Importancia biológica}

Para estimar la importancia biológica, combinamos la diversidad local, derivada a partir de los Índices Dinámicos de Hábitat (IDH), y la heterogeneidad climática, como indicador de la variación en la composición biológica. Estos índices han sido usados para describir patrones globales de biodiversidad; sin embargo, a escala de paisaje pueden presentar sesgos, ya que pueden no reflejar apropiadamente los cambios en diversidad. No obstante, la escasez de muestreos en el área de estudio no permite realizar validaciones de los IDH a esta escala. Por otro lado, los IDH son un proxy para conocer los cambios en diversidad a lo largo del tiempo a diferentes escalas espaciales, y son útiles para evaluaciones rápidas de la representatividad y efectividad de las $\mathrm{AP}$ (Hobi et al., 2017; Hobi et al., 2021; Politi et al., 2021).

Primero, utilizamos los IDH como una aproximación de la biodiversidad en el COA (Radeloff et al., 2019), los cuales fueron obtenidos del repositorio SILVIS LAB para el promedio de los años 2003 a 2014, con base en el Indice de Vegetación de Diferencia Normalizada (NDVI, por sus siglas en inglés) en formato ráster con una resolución espacial de $1 \mathrm{~km} \times 1 \mathrm{~km}$. Los IDH permiten conocer la riqueza de especies, basados en medidas de productividad bajo los siguientes supuestos (Radeloff et al., 2019): 1) la diversidad de especies aumenta con la productividad (hipótesis de energía; Wright, 1983); $\mathrm{IDH}_{1}$ estimado como la sumatoria de la productividad anual según el NDVI; 2) disminuye hacia los ambientes extremos (hipótesis de filtro ambiental; Currie et al., 2004); $\mathrm{IDH}_{2}$ estimado como el valor mínimo anual del NDVI; y 3) aumenta con la estabilidad ambiental (hipótesis de estacionalidad; Janzen, 1967); $\mathrm{IDH}_{3}$ estimado como la desviación estándar anual del NDVI. Estos tres índices son complementarios entre sí y proporcionan una mirada integral de la riqueza de especies de una región. Para realizar posteriores análisis, se hizo una división del COA en cuadrantes de $1 \mathrm{~km} \times 1 \mathrm{~km}$ y se extrajo el valor de cada uno de los IDH para cada cuadrante.

Segundo, estimamos la heterogeneidad climática a partir de las variables bioclimáticas (i.e., temperatura media anual, precipitación anual, estacionalidad de la temperatura, precipitación del cuarto más seco y variabilidad de la precipitación) de WorldClim (Fick \& Hijmans, 2017) a una resolución espacial de $1 \mathrm{~km} \times 1 \mathrm{~km}$. A partir de esta información, estimamos tres grupos climáticos para la región de estudio, a través del método de aglomeración conocido como k-medias. Este método establece grupos con varianzas homogéneas distinguibles de otros grupos (Altman \& Krzywinski, 2017; Gardner et al., 2020). Finalmente, para validar los grupos climáticos, realizamos comparaciones de la composición de especies de aves, mamíferos y angiospermas entre grupos, usando listados de especies derivados de los registros depositados en el Repositorio Mundial de Información sobre Biodiversidad (GIBIF, por sus siglas en inglés) (Beck et al., 2014) y cuantificamos el porcentaje de similitud entre ellos (i.e., Diversidad Beta, número de especies compartidas/la suma de especies entre los dos sitios; Krebs, 1999).

Por último, calculamos el índice de importancia biológica ubicando los sitios con mayores valores del $\mathrm{IDH}_{1}$ dentro de cada grupo climático, para localizar las áreas de mayor interés. Con el fin de establecer comparaciones entre los grupos climáticos, se escalaron los $\mathrm{IDH}_{1}$ de cada grupo por su mayor valor; de esta forma todos varían entre 0 y 1 , siendo 1 los sitios con mayor importancia biológica (i.e., píxeles con mayor riqueza de especies).

\section{Vulnerabilidad}

Se estimó la vulnerabilidad a la pérdida de hábitat y conectividad por medio de la cuantificación de la deforestación y la fragmentación. La cuantificación de la deforestación y la fragmentación de los bosques dentro y fuera de las AP se realizó usando información satelital derivada de Landsat (Hansen et al., 2013). Los datos de deforestación fueron obtenidos de los resultados del análisis de series de tiempo de imágenes Landsat realizado por Hansen et al. (2013), depositados en el geoportal Global Forest Change. La capa ráster utilizada fue Year of gross forest cover loss event (i.e., lossyear), la cual tiene una resolución espacial aproximada de $30 \mathrm{~m} \times 30 \mathrm{~m}$ y proporciona información sobre eventos de deforestación anual. El ráster de deforestación fue transformado en 
unos y ceros, donde uno representa un evento de deforestación para cualquier año, y cero, ningún evento de deforestación. Se utilizaron los cuadrantes de $1 \mathrm{~km} \times 1$ $\mathrm{km}$ para extraer la suma de los pixeles deforestados para cada uno de estos cuadrantes, lo cual nos permitió conocer el valor de los IDH en cada sitio que ha sido deforestado y en los que no ha sido deforestado. Finalmente, con el uso del vector de AP para el COA y el ráster de deforestación, extrajimos la deforestación dentro de las AP y por fuera de ellas. La transformación del ráster de deforestación fue realizada utilizando las funciones de los paquetes Raster y rgdal del programa R.

Para estimar la fragmentación, en primer lugar, obtuvimos el ráster de cobertura de bosque para el año 2019, calculado a partir de un ráster de cobertura del año 2000 (i.e., ráster de referencia) obtenido de la base de datos de Global Forest Change (treecover2000; Hansen et al., 2013). El ráster de referencia tiene una resolución espacial de aproximadamente $30 \mathrm{~m} \times 30 \mathrm{~m}$ por píxel y contiene valores de 0 a $100 \%$ de cobertura. Los valores del ráster de referencia fueron transformados a cero y uno, donde los píxeles con cobertura de bosque mayores o iguales a $80 \%$ tomaron valores de uno. Posteriormente, sustrajimos los píxeles que fueron deforestados (i.e., ráster anteriormente obtenido) desde el año 2001 hasta el año 2019. El análisis fue realizado utilizando las funciones del paquete Raster del programa R.

El índice de fragmentación $f$ en el COA se calculó a partir del ráster de cobertura, contando el número de píxeles que contiene bosque (i.e., píxeles iguales a $1, n$ ); el número de grupos (i.e., uno o más píxeles continuos con bosque, g); el número de píxeles conectados (i.e., aquellos que tienen más de dos píxeles aledaños con cobertura boscosa, c); y el número de píxeles totales $(N)$, dentro de una ventana de análisis de $3 \mathrm{~km} \times 3 \mathrm{~km}$ (Ecuación 1). Este índice tiene valores de cero cuando la fragmentación es alta y valores cercanos a uno cuando la fragmentación es nula (González-Caro \& Vásquez, 2017). El índice fue obtenido utilizando las funciones de los paquetes $s p$, raster y maptools del programa R.

$$
f=\frac{n c+n}{g N^{2}} \quad \text { Ecuación } 1
$$

$\mathrm{Al}$ igual que con el ráster de deforestación, para la fragmentación se utilizaron los cuadrantes de $1 \mathrm{~km} \times 1 \mathrm{~km}$ para extraer el valor de la fragmentación para cada uno de estos cuadrantes. Esto nos permite conocer el valor de los IDH y su grado de fragmentación en un área específica. Finalmente, con el uso del vector de AP para el COA y el ráster de fragmentación extrajimos la fragmentación dentro de las AP y por fuera de ellas.

\section{Análisis estadísticos}

Para analizar si las áreas de mayor importancia biológica en el COA están representadas en las AP, consideramos las metas de conservación reconocidas globalmente bajo el Convenio de Diversidad Biológica (CDB). Se esperaba que para el año 2020 al menos $17 \%$ de la tierra fuera declarada como AP (meta Aichi 12). Sin embargo, diferentes estudios han demostrado que solo $17 \%$ del territorio puede ser insuficiente para mantener poblaciones viables de muchas especies, por lo cual se ha sugerido incrementar el área hasta 30 \% aproximadamente (Venter et al., 2018). De esa manera, calculamos si las áreas con mayor importancia biológica están representadas en al menos $17 \%$ y $30 \%$ en las AP del COA. Para esto ordenamos los valores del mayor al menor y seleccionamos los píxeles correspondientes al $17 \%$ y $30 \%$ del área de estudio, desde el valor más alto para cada uno de los tres grupos climáticos.

Para evaluar el efecto de la deforestación y la fragmentación dentro del COA, comparamos los IDH obtenidos entre 2003 y 2014 entre áreas deforestadas (durante 2014 y 2019) y áreas no deforestadas. De igual forma se hizo para áreas fragmentadas y no fragmentadas. Para esto utilizamos la prueba de Kolmogórov-Smirnov, la cual nos permite comparar distribuciones no paramétricas. Para realizar la prueba, utilizamos la función ks.test del paquete stats del programa R (Lopes et al., 2007; Young, 1977). Este análisis se realizó separando AP y áreas no protegidas, donde los valores perdidos de bosque (en hectáreas) dentro de las AP significan deficiencia en la protección y manejo de dichas áreas y fuera de ellas indica pérdida de conectividad regional. Todos los análisis fueron ejecutados usando R 3.5.1.

\section{Resultados}

El área de cobertura boscosa actual en el COA fue de $2720.6 \mathrm{~km}^{2}$, es decir, $\sim 65.24 \%$ de su extensión total son bosques (Figura S1c). De estos, aproximadamente $1265.25 \mathrm{~km}^{2}$ (30,34 \% de los bosques actuales y el 40.21 $\%$ del área total) se encuentran dentro de alguna figura de protección (Tabla S1).

\section{Importancia biológica}

En promedio, la productividad anual acumulativa (i.e., $\mathrm{IDH}_{1}$, derivado del NDVI banda 1) fue de $15.04 \pm 3.30$, 
y el $65.42 \%\left(\mathrm{~km}^{2}\right)$ del COA presentó valores mayores o iguales a 15, es decir, áreas de alta productividad y diversidad de especies. Por otro lado, la productividad anual mínima (i.e., $\mathrm{IDH}_{2}$, derivado del NDVI banda 2) fue $0.42 \pm$ 0.23 , con solo el $29.84 \%$ del corredor presentando valores de alta diversidad de especies. Finalmente, la variación estacional de la productividad, (i.e., $\mathrm{IDH}_{3}$, derivado del NDVI banda 3) fue $0.19 \pm 0.17$, donde $\sim 86 \%$ del corredor tuvo valores menores o iguales a 0.2 , lo cual significa una elevada diversidad de especies en casi toda su extensión.
La heterogeneidad climática del COA nos permitió diferenciar tres zonas climáticas (grupos 1, 2 y 3; Figura S1b). El grupo 2 fue el de mayor extensión con $\sim 1828.66 \mathrm{~km}^{2}$, seguido del grupo $3\left(1593.68 \mathrm{~km}^{2}\right) \mathrm{y}$, por último, el grupo $1\left(745.70 \mathrm{~km}^{2}\right)$. Aproximadamente el $45.32 \%$ de la extensión del grupo 3 estuvo dentro de un área protegida, a diferencia de los otros dos grupos que tuvieron una menor representación de AP dentro de sus límites, $37.03 \%$ y $36.09 \%$, grupo 1 y grupo 2, respectivamente.
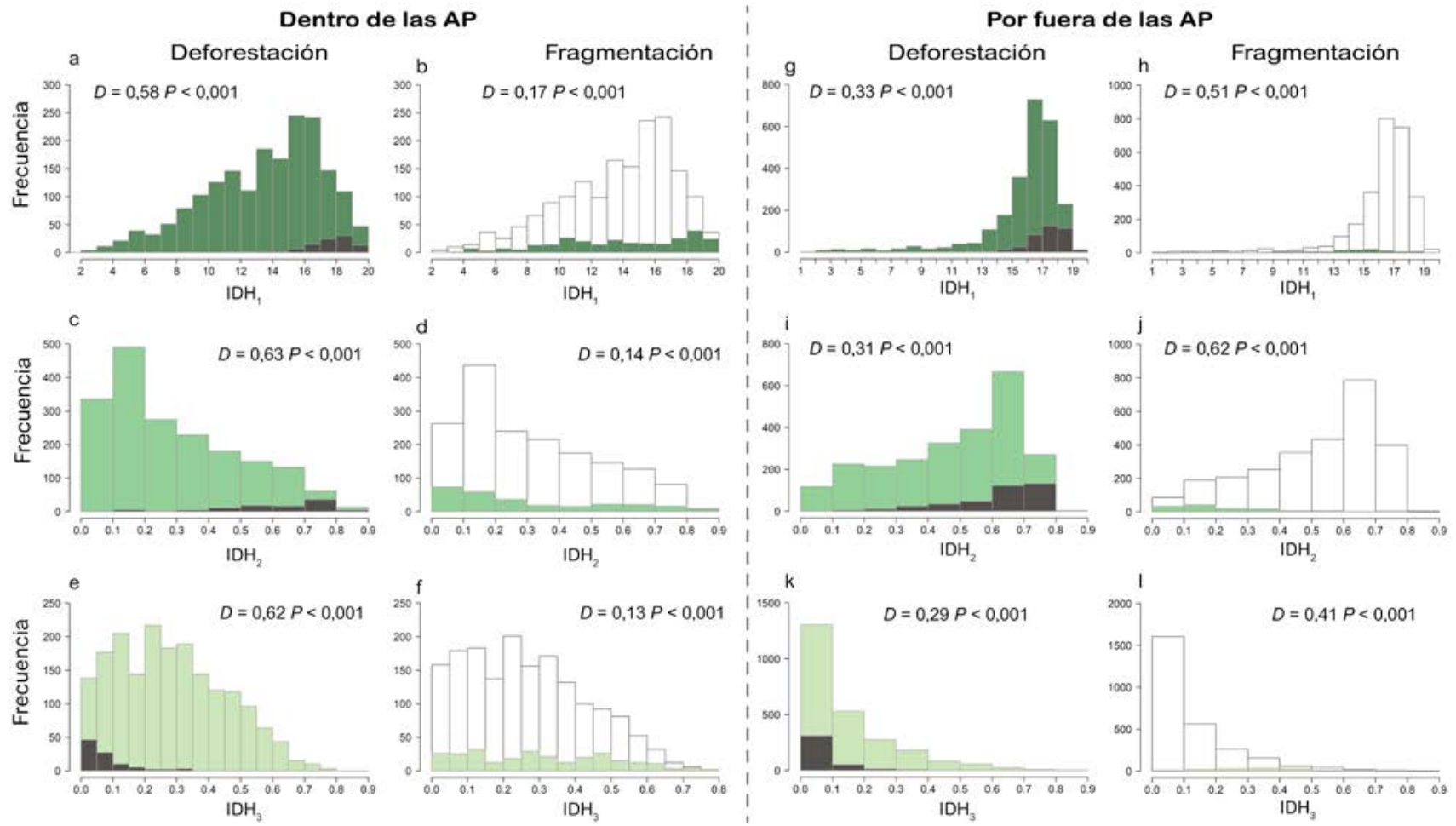

Figura 1. Distribución de los Índices Dinámicos de Hábitat en sitios deforestados y fragmentados dentro y fuera de las AP del Corredor del Oso de Anteojos (COA), en Antioquia, Colombia. Las gráficas enseñan los histogramas de distribución de los Índices Dinámicos de Hábitat ( $\mathrm{IDH}_{1}, \mathrm{IDH}_{2}, \mathrm{IDH}_{3}$; barras de color verde), donde $a, b, c, d, g, h, i$ y $j$ corresponden a los $\mathrm{IDH}_{1} \mathrm{y}$ $\mathrm{IDH}_{2}$, en los cuales, a medida que aumenta el valor del índice, hay mayor diversidad de especies; $\mathrm{y} e, f, k$ y $l$ corresponden al $\mathrm{IDH}_{3^{\prime}}$ donde a mayor valor, menor diversidad de especies. Los valores de cada índice en los sitios deforestados entre $2001 \mathrm{y}$ 2019 se representan en las barras grises, es decir, el valor de los IDH para cada sitio que fue deforestado. Las barras de color blanco representan los sitios con alta fragmentación por fuera y dentro de las AP, es decir, el valor de los IDH en los sitios con alta fragmentación (<0.7). D es el estadístico de la prueba de Kolmogórov-Smirnov.

Figure 1. Distribution of Dynamic Habitat Indices in deforested and fragmented sites inside and outside the Spectacled Bear Corridor (COA) protected areas in Antioquia, Colombia. Graphs show the distribution histograms of the Dynamic Habitat Indices (HDI1, HDI2, HDI3; green bars) where $a, b, c, d, g$, h, i and j correspond to HDI1 and HDI2, in which as the index value increases, there is greater species diversity; and e, $\mathrm{f}, \mathrm{k}$ and $\mathrm{l}$ correspond to HDI3, as the value increases, the species diversity decreases. The values of each index in deforested sites between 2001 and 2019 are represented in gray bars, i. e., the HDI value for each site that was deforested. White bars represent sites with high fragmentation outside and inside protected areas, i. e., the HDI value at sites with high fragmentation (< 0.7). D is the Kolmogórov-Smirnov test statistic. 
En cuanto a las comparaciones de la composición de especies entre los tres grupos climáticos, encontramos que estos se diferencian principalmente en su composición de especies de plantas (angiospermas). Como era de esperarse, los grupos taxonómicos con mayor movilidad como aves o mamíferos son muy similares, pues el área del COA es relativamente pequeña. Sin embargo, los grupos climáticos 2 y 3 se diferencian en la composición de mamíferos. Es importante resaltar que el grupo climático 2 es el que tiene un mayor número de registros biológicos, mientras que los otros están pobremente estudiados, lo que reduce la confiabilidad de la comparación de los listados.

Por último, los sitios con mayores valores de importancia biológica para cada grupo climático tuvieron valores de $0.889 \pm 0.05,0.883 \pm 0.04$ y $0.885 \pm 0,05$, respectivamente (Figura S1c). Aproximadamente el $49.80 \%$, $64.06 \%$ y $44.29 \%$ de cada grupo climático, en orden, representan los sitios más relevantes (i.e., valores cercanos a 1) para conservar.

\section{Vulnerabilidad}

En los últimos 19 años, el COA perdió en cobertura boscosa alrededor de $162.37 \mathrm{~km}^{2}\left(8.55 \pm 5.94 \mathrm{~km}^{2}\right.$. año ${ }^{-1}$ ), siendo el 2017 el año que presentó una deforestación cuatro veces más grande que el promedio anual. Específicamente, la deforestación por dentro y fuera de las AP del COA fue de $37.93 \mathrm{~km}^{2}$, equivalente al $2.2 \%$ del área total protegida $\left(0.22 \pm 0.36 \mathrm{~km}^{2}\right.$. año-1; Figura 1a, c y e; Tabla S1) y $124.43 \mathrm{~km}^{2}, 4.9 \%$ del área $\left(6.55 \pm 4.20 \mathrm{~km}^{2}\right.$.año-1; Figura $1 \mathrm{~g}$, i y k), respectivamente. El área protegida con mayor tasa de deforestación $\left(\sim 0.64 \mathrm{~km}^{2}\right.$.año $\left.{ }^{-1}\right)$ fue la RFPN Farallones del Citará ubicada en el suroccidente del COA. Es importante resaltar que la deforestación estuvo fuertemente correlacionada con la extensión de las $\mathrm{AP}(r=0.63, P<0.01)$. Es decir, a mayor área se evidenció un incremento proporcional. Adicionalmente, la deforestación, tanto dentro de las AP como por fuera de ellas se dio en los sitios de mayor riqueza de especies, como lo confirma la prueba de Kolmogórov-Smirnov $(P<0.001)$ (Figura 1a, e, g, i y k).

En cuanto a la fragmentación, solo el $4.27 \%$ de la cobertura boscosa por fuera de las AP presentó bajo grado de fragmentación (i.e., valores entre 0.8 y 1.0; Figura S1d), principalmente en el centro y noreste del COA y en lugares con baja riqueza de especies. De igual forma, dentro de las AP, solo el $16 \%$ de la cobertura boscosa presentó valores bajos de fragmentación. La fragmentación en el área restante (i.e., $84 \%$, valores entre 0.0 y 0.7 ) se dio en los sitios con mayor riqueza de especies (prueba de Kolmogórov-Smirnov $P<0.001$; Figura 1b, d, f, h, j, l) lo que favorece al aislamiento de las AP. Por otra parte, encontramos 26 remanentes de bosque sin ningún grado de fragmentación en el COA, de los cuales, el $72.50 \%$ se encuentran en las AP (Figura S1d). Es de resaltar que más del $50 \%$ de los remanentes de bosque tienen pendientes mayores a $20^{\circ}$ (de fuertemente inclinadas a escarpadas). Los remanentes poseen áreas entre $99.36 \mathrm{~km}^{2}$ y $0.33 \mathrm{~km}^{2}$ y están distribuidos en su mayoría en el noroeste del COA $(57.70 \%$ de ellos) dentro de las AP PNN Las Orquídeas, RFPN de Urrao y RFPN Carauta.

\section{Representación dentro de las AP}

Las AP representan el $40.21 \%$ del área total del COA. No obstante, al evaluar la representatividad de las áreas de mayor importancia biológica para cada grupo climático dentro de las AP del COA, según los objetivos de conservación mundial (17 \% meta Aichi 12 y $30 \%$ ), identificamos que los grupos climáticos no están apropiadamente representados por las AP dentro del COA (i.e., ningún grupo alcanza el $100 \%$ para cumplir con los objetivos de conservación mínimos $17 \%$ y $30 \%$; Tabla 1). El grupo menos representado es el uno (menos del $15 \%$ en ambos casos), el cual está distribuido hacia la vertiente occidental (Chocó biogeográfico). Los otros dos grupos se encuentran representados en aproximadamente la mitad de sus áreas prioritarias. Aunque el PNN Las Orquídeas no está completamente incluido en el COA, el aporte de este no es significativo para el área de estudio, comparado con otras figuras de conservación. Las reservas forestales de Carauta, Urrao y Farallones de Citará tienen un aporte importante, contribuyendo a la conservación de los grupos climáticos simultáneamente, con valores significativos (Tabla 1). Los DMI tienen mayor impacto en la conservación del $30 \%$ que del $17 \%$, lo que implica que están localizados hacia áreas de menor importancia que los otros esquemas de conservación. La representatividad de las reservas de la sociedad civil es baja, debido a su poca área, comparada con otras figuras de conservación. 
Tabla 1. Porcentaje de representatividad de las áreas de importancia biológica para cada grupo climático, dentro de las de AP del Corredor del Oso de Anteojos (COA), en Antioquia, Colombia, según los objetivos de conservación mundial (17 \% meta Aichi 11 y 30 \%). En negrilla están resaltados los valores más altos por grupo y objetivo de conservación. DRMI: Distrito Regional de Manejo Integrado; PNN: Parque Nacional Natural; PNR: Parques Naturales Regionales; RFPN: Reserva Forestal Protectora Nacional, RNSC: Reserva Natural de la Sociedad Civil. El total representa el porcentaje de cumplimiento de la meta propuesta.

Table 1. Biological important areas representativity percentage for each climate group within the Spectacled Bear Corridor (COA) protected areas in Antioquia, Colombia, according to global conservation objectives (17 \% Aichi 11 target, and $30 \%)$. The highest values per group and conservation target are highlighted in bold. DRMI: Regional Integrated Management District; NNP: National Natural Park; RNP: Regional Natural Parks; RFPN: National Protected Forest Reserve; RNSC: Civil Society Natural Reserve.

\begin{tabular}{|c|c|c|c|c|c|c|}
\hline \multirow[b]{2}{*}{ Áreas protegidas } & \multicolumn{3}{|c|}{$17 \%$} & \multicolumn{3}{|c|}{$30 \%$} \\
\hline & $\underset{(\%)}{\text { Grupo } 1}$ & $\begin{array}{c}\text { Grupo } 2 \\
(\%)\end{array}$ & $\begin{array}{c}\text { Grupo } 3 \\
(\%)\end{array}$ & $\begin{array}{c}\text { Grupo } 1 \\
(\%)\end{array}$ & $\underset{(\%)}{\text { Grupo } 2}$ & $\begin{array}{c}\text { Grupo } 3 \\
(\%)\end{array}$ \\
\hline DRMI Alto del Insor & - & 3.89 & - & - & 7.89 & - \\
\hline $\begin{array}{l}\text { DRMI Cuchilla Cerro Plateado Alto San } \\
\text { José }\end{array}$ & 4.76 & - & 0.32 & 8.49 & 0.00 & 1.45 \\
\hline DRMI Cuchilla Jardín Támesis & - & 1.39 & 1.92 & - & 2.21 & 10.73 \\
\hline PNN Las Orquídeas &.-- & 4.72 & 9.62 & - & 6.78 & 10.55 \\
\hline PNR Corredor de las Alegrías & - & 4.17 & 0.00 & - & 7.57 & 1.27 \\
\hline RFPN Carauta & - & 6.11 & 29.49 & 0.77 & 5.84 & 21.82 \\
\hline RFPN de Urrao & - & 17.22 & 2.24 & - & 16.09 & 3.27 \\
\hline RFPN Farallones del Citará & 4.08 & - & 2.24 & 5.41 & - & 3.45 \\
\hline RNSC Buena Vista* & - & - & - & - & - & - \\
\hline RNSC Colibrí del Sol & - & - & - & - & 0.16 & - \\
\hline RNSC de las Aves Colibrí del Sol* & - & - & - & - & - & - \\
\hline RNSC La Violeta* & - & - & - & - & - & - \\
\hline RNSC Orquídeas * & - & - & - & - & - & - \\
\hline Total (\%) & 8.84 & 37.50 & 45.83 & 14.67 & 46.53 & 52.55 \\
\hline
\end{tabular}

* AP con una extensión considerablemente pequeña que no alcanzan a proteger áreas de importancia biológica de manera representativa.

\section{Discusión}

A partir de información derivada de sensores remotos y grandes bases de datos, logramos realizar una identificación rápida de la distribución de la perdida de cobertura vegetal, fragmentación de los bosques y su relación sobre la diversidad en el COA en Antioquia. El COA, ubicado en una de las últimas regiones relativamente mejor conservadas en el departamento de Antioquia, con un gran auge en la consolidación de diversas organizaciones ambientales locales y regionales (Corporación Gaia, VerdeAgua, Nodo de reservas de la sociedad Civil, Corantioquia, entre otras), presenta una de las coberturas de bosque más altas del departamento (65\% COA vs el 58\% Antioquia, Morales et al., 2020).
Por tanto, se están conservando los bosques al compararlo con otras zonas en el departamento, como en las subregiones del Bajo Cauca, Nordeste y Magdalena Medio, donde se encuentran los municipios con mayor deforestación en Antioquia y donde se ha deforestado entre los años 2000 y 2019 aproximadamente $3000 \mathrm{~km}^{2}$ (Morales et al., 2020), 18 veces más que la deforestación acumulada en el COA $\left(162.37 \mathrm{~km}^{2}\right)$. No obstante, el COA no es ajeno a los motores de transformación del paisaje que enfrenta la región. Si bien presenta una tasa de deforestación relativamente baja para el departamento de Antioquia con solo 4\% de pérdida acumulada en los últimos 19 años, los procesos de fragmentación son altos (i.e. $\sim 90 \%$ de la cobertura boscosa presenta valores entre 0.0 y 0.7 ) y las coberturas boscosas continuas 
grandes son escasas. Sumado a que los procesos de fragmentación se concentran en los sitios con mayor importancia biológica (Figura 1b, f, h, j y l).

Adicional a la consecuente pérdida de hábitat, la deforestación en sí misma genera una mayor sensibilidad a las perturbaciones al interior de los remanentes de bosque, tales como incendios forestales y tala selectiva, lo que a su vez induce a cambios en la estructura del bosque y la composición de especies (Barlow et al., 2016). Igualmente, aunque no existe un consenso sobre los efectos de la fragmentación sobre la biodiversidad (Haddad et al., 2015; Piquer-Rodríguez et al., 2015), varios estudios han encontrado que ésta afecta negativamente la riqueza de especies, la retención de nutrientes y las dinámicas tróficas de los ecosistemas (Zhai et al., 2014; Haddad et al., 2015; Piquer-Rodríguez et al., 2015). Por tanto, de no mitigarse la fragmentación de los bosques, podríamos enfrentar procesos graves de defaunación en una de las últimas regiones conservadas del departamento.

\section{¿Se están conservando los bosques y las áreas con ma- yor biodiversidad en el Corredor del Oso de Anteojos en la Cordillera Occidental de Antioquia?}

Sorpresivamente, el sistema de AP dentro de la región ( $\sim 0 \%$ del área total del COA) no representa de manera apropiada su importancia biológica. Es decir, el $17 \%$ del territorio con mayor concentración potencial de especies no se encuentra cubierto por el sistema de AP. Al analizar cada uno de los grupos climáticos obtenidos, ninguno de ellos se encuentra representado en las $\mathrm{AP}$, al menos en la mitad de lo esperado (Tabla 1). En particular, uno de los grupos climáticos que contienen la vertiente occidental de la cordillera Occidental en el COA (Chocó biogeográfico; Figura S1b) está representado en las AP en menos del $15 \%$ (2.55\% del territorio). Otros ecosistemas, como los bosques altoandinos y páramos, los cuales se encuentran en los otros dos grupos climáticos, están mejor representados en el sistema de AP.

La biodiversidad del Chocó biogeográfico en Antioquia, vertiente occidental de la Cordillera Occidental, es altamente desconocida. Para esta área, el número de registros reportado en GBIF corresponde en su gran mayoría a aves (probablemente el grupo taxonómico más estudiado) con solo 45 registros, lo cual demuestra el vasto desconocimiento que se tiene de esta región. Sin embargo, es conocido que la Cordillera Occidental, y probablemente su vertiente occidental, está entre las regiones con mayor endemismo del mundo (Kattan et al., 2004; Sandel et al., 2011). Si a esto sumamos la escasa representatividad de esta región en las AP del COA y en general del departamento de Antioquia, se puede concluir que esta zona debería ser una de las principales prioridades de conservación en Antioquia, y merecería ser incluida en la planificación territorial a nivel del COA y departamental. También, algunas áreas climáticamente similares a los páramos en la cuchilla de Jardín-Támesis podrían incluirse como ecosistemas estratégicos, para incrementar la eficacia en su conservación y reforzar el sistema de AP dentro del COA.

Por otro lado, detectamos procesos de desforestación y fragmentación en las AP dentro del COA (Figura 1a, c y e; Figura S1b). Es importante resaltar que la pérdida y degradación del hábitat converge con las áreas donde los IDH muestran una mayor riqueza de especies potencial (Figura 1a-f), lo cual puede estar relacionado al uso de las áreas con condiciones óptimas para la producción agrícola o ganadera. Estos cambios en el uso del suelo son permitidos en algunas de las estrategias de conservación que involucran desarrollo sostenible, como los DMI. Sin embargo, en AP estrictas, como los PNN, también encontramos registros de deforestación (Tabla S1). Lo anterior, ha sido mostrado a escala nacional, y Clerici et al. (2020) reportaron pérdida de bosques en el sistema de parques nacionales en los últimos años, atribuido a los cambios en los territorios relacionados, entre otros a procesos de acaparamiento de tierra que se han generado posterior a la firma del Acuerdo de Paz de Colombia en los años 2016 y 2017. De igual manera, a nivel departamental y en el COA, observamos un incremento significativo en la deforestación para este periodo (Morales et al., 2020).

Tanto la pérdida de bosque como la fragmentación son mayores en las zonas por fuera de las AP del COA (Figura 1g-1; Figura S1b y c). Sin embargo, la fragmentación tiene un mayor impacto en las regiones por fuera de las AP. La fragmentación se midió a través del grado de cohesión de los píxeles de bosque dentro de una ventana de $3 \mathrm{~km} \times 3 \mathrm{~km}$, donde se observa que la mayoría del área del COA no contiene bosques continuos de este tamaño, es decir, gran parte de los bosques fuera del sistema de AP son fragmentos de menos de $1 \mathrm{~km}^{2}$ (100 ha; Figura S1d). En términos de la viabilidad de las especies, se puede pensar que estos pequeños parches de bosque no son importantes, pero se ha demostrado que, en los Andes, estos son claves para mantener relictos de poblaciones de especies endémicas o raras, y además facilitan la conectividad entre grandes áreas 
de bosque (Wilson \& Rhemtulla, 2018). Es por ello que mantenerlos, y preferiblemente incrementarlos, es un asunto prioritario. Adicionalmente, estos fragmentos pueden tener un valor agregado en la agricultura local (Ricketts et al., 2004), manteniendo poblaciones de polinizadores (González-Chaves et al., 2020). Por lo cual, intensificar el estudio de las poblaciones en estos fragmentos y sus efectos en la conectividad de las poblaciones naturales es una línea de investigación clave para el desarrollo territorial sostenible.

\section{Perspectivas y limitaciones}

La importancia biológica que implementamos, basada en la combinación de grupos climáticos y los IDH, nos permitió, de manera relativamente fácil, hacer una evaluación preliminar del estado de los bosques y su representatividad el área de estudio, y su dinámica entre 2003 y 2019. Así mismo, nos posibilitó identificar que las áreas de pérdida en este periodo han sido áreas importantes para la conservación, probablemente con un mayor valor que áreas conservadas actualmente. Adicionalmente, la delimitación de los grupos climáticos nos permitió maximizar la cobertura de diferentes ecosistemas dentro del área de estudio de manera sistemática y enfocada al contexto biogeográfico de la misma. Es decir, que al diferenciar en el COA áreas similares climáticamente, nos pudimos enfocar en la cobertura de cada área y realizar un análisis por separado para cada grupo. Aunque esta metodología puede ser poco precisa en generar regiones bióticamente homogéneas, como suponemos, puede ser muy útil en áreas con escasa información de su biodiversidad, como es el caso del $\mathrm{COA}$, o de muchas regiones del trópico en general; no obstante, es importante calibrar estos resultados con estudios de línea base de biodiversidad (Arbeláez-Cortés et al., 2017). Estos grupos climáticos fueron validados mediante la comparación de listas de especies derivadas de GBIF, soportando que los grupos climáticos faciliten la identificación de grupos bióticamente homogéneos. Sin embargo, la validación de nuestros resultados con muestreos en campo y sitios de monitoreo constante nos pueden permitir calibrar de manera apropiada los IDH para futuros estudios de manera más precisa.

Aunque actualmente podemos conocer los procesos de deforestación, idealmente se deberían incluir predicciones futuras del comportamiento de la pérdida de hábitat para tener un escenario más amplio de los cambios que puede tener una región (Morelli et al., 2020). No obstante, los modelos para realizar predicciones de deforestación en escenarios futuros pueden tener diferentes limitaciones (Morelli et al., 2020). Por otra parte, además de medir el efecto de la deforestación y la fragmentación, es importante incluir el efecto que podría tener el cambio climático y sus sinergias sobre los ecosistemas, puesto que solamente el efecto del cambio climático podría reducir drásticamente el hábitat de grandes especies sombrilla, como el Oso de Anteojos.

Hemos incorporado en este análisis dos variables principales para que sean incluidos al sistema de conservación actual del departamento: representatividad de las áreas de mayor importancia biológica en las AP, incluyendo la biodiversidad, y la evaluación de la vulnerabilidad basado en fragmentación y deforestación, puesto que el COA busca proteger una amplia variedad de ecosistemas en los cuales habita el oso de anteojos. Las acciones de protección buscan maximizar la biodiversidad protegida, su integridad (áreas amplias con poca fragmentación) y que estas acciones incluyan las variaciones en diversidad (representatividad). Probablemente hemos excluido análisis minuciosos que requieren corroboración en campo, pero ante la realidad de nuestras regiones, en las cuales los recursos para realizar inventarios y monitores de la biodiversidad son escasos, consideramos que el uso de sistemas de información geográfico puede guiar los esfuerzos para priorizar exploraciones en áreas concretas y así avanzar en nuevas delimitaciones. Por otro lado, si bien nuestros resultados nos señalan la debilidad de las AP en el COA en proteger la cobertura vegetal, no obstante, es la estrategia más efectiva a nivel mundial. Sin embargo, en aquellas AP donde los niveles de vulnerabilidad son más altos, se podría considerar incrementar las restricciones. Ahora bien, como lo señalan Corantioquia y la Corporación Gaia, la conservación de este territorio debe incorporar múltiples opciones de desarrollo sostenible, que incluyan procesos de educación y desarrollo económico integrados, como, por ejemplo, el programa Abrazando Montañas, sumado al acompañamiento de las múltiples iniciativas locales, para garantizar la conservación del territorio de la mano de sus habitantes (Molina \& Aristizábal, 2018).

\section{Conclusión}

Aunque el área del Corredor del Oso de Anteojos se encuentra en un $40 \%$ dentro de AP, éstas no necesariamente fueron diseñadas para maximizar la protección de las especies en la región. Por esto, es una prioridad incluir nuevas áreas, principalmente en la vertiente occidental de esta cordillera (Chocó biogeográfico). También se 
encontraron pérdidas de bosque dentro de las AP restrictivas, como los parques nacionales, lo cual hace un llamado a los entes administrativos a incrementar las estrategias para reducirla, ya que, si bien la deforestación es relativamente menor en las $\mathrm{AP}$, los procesos de fragmentación son significativos, es decir, que los motores de trasformación permean las AP. Finalmente, se identificó que la fragmentación y pérdida de pequeños parches a lo largo del corredor puede disminuir sustancialmente la conectividad entre los parches $\mathrm{y}$, por ende, contravenir el objetivo principal de esta iniciativa de conservación de mantener las poblaciones de osos de anteojos. En ese sentido, es relevante ejecutar acciones que aumenten la efectividad de las AP y sus alrededores, tales como programas de compensación a comunidades locales, los cuales han demostrado ser efectivos contra la explotación forestal y la deforestación en los bosques tropicales.

\section{Agradecimientos}

Agradecemos a Sebastián González, Dubán Canal y Álvaro Idárraga por sus comentarios al texto, y a Valeria Zapata por la revisión de estilo. Agradecemos al comité técnico del Observatorio de Bosques de Antioquia y al Programa de Bosques Andinos, parte del Programa Global de Cambio Climático de la Agencia Suiza para el Desarrollo y la Cooperación (COSUDE).

\section{Referencias}

Altman, N. \& Krzywinski, M. (2017). Points of significance: Clustering. Nature Methods, 14(6), 545-546. https:/ / doi.org/10.1038/nmeth.4299

Andam, K. S., Ferraro, P. J., Pfaff, A., Sánchez-Azofeifa, G. A. \& Robalino, J. A. (2008). Measuring the effectiveness of protected area networks in reducing deforestation. Proceedings of the National Academy of Sciences of the United States of America, 105(42), 1608916094.

https:/ / doi.org/10.1073/pnas.0800437105

Arbeláez-Cortés, E., Acosta-Galvis, A. R., DoNascimiento, C., Espitia-Reina, D., González-Alvarado, A. \& Medina, C. A. (2017). Knowledge linked to museum specimen vouchers: measuring scientific production from a major biological collection in Colombia. Scientometrics, 112(3), 1323-1341. https:/ / doi.org/10.1007/s11192-017-2461-4

Armenteras, D., Gast, F. \& Villareal, H. (2003). Andean forest fragmentation and the representativeness of protected natural areas in the eastern Andes, Colombia. Biological Conservation, 113(2), 245-256. https:/ / doi.org/10.1016/S0006-3207(02)00359-2 Banco Mundial. (2013). Andean population. Recuperado de data.worldbank.org

Barlow, J., Lennox, G. D., Ferreira, J., Berenguer, E., Lees, A. C., Nally, R. Mac, ... Gardner, T. A. (2016). Anthropogenic disturbance in tropical forests can double biodiversity loss from deforestation. Nature, 535(7610), 144-147.

https:// doi.org/10.1038/nature18326

Beck, J., Böller, M., Erhardt, A. \& Schwanghart, W. (2014). Spatial bias in the GBIF database and its effect on modeling species' geographic distributions. Ecological Informatics, 19, 10-15. https:/ / doi.org/10.1016/j.ecoinf.2013.11.002

Brooks, T. M., Mittermeier, R. A., Da Fonseca, G. A. B., Gerlach, J., Hoffmann, M., Lamoreux, J. F., ... Rodrigues, A. S. L. (2006). Global biodiversity conservation priorities. Science, 313(58), 58-61. https:// doi.org/10.1126/science.1127609

Bruner, A. G., Gullison, R. E., Rice, R. E. \& Da Fonseca, G. A. B. (2001). Effectiveness of parks in protecting tropical biodiversity. Science, 291(5501), 125-128. https:// doi.org/10.1126/science.291.5501.125

Cardinale, B. J., Duffy, J. E., González, A., Hooper, D. U., Perrings, C., Venail, P., ... Naeem, S. (2012). Biodiversity loss and its impact on humanity. Nature, 486(7401), 59-67. https://doi.org/10.1038/nature11148

CBD-Secretariat of the Convention on Biological Diversity. (2004). Programme of Work on Protected Areas. Convention on Biological Diversity.

Clerici, N., Armenteras, D., Kareiva, P., Botero, R., Ramírez-Delgado, J. P., Forero-Medina, G., ... Biggs, D. (2020). Deforestation in Colombian protected areas increased during post-conflict periods. Scientific Reports, 10(1), 1-10. https:// doi.org/10.1038/s41598-020-61861

Currie, D. J., Mittelbach, G. G., Cornell, H. V., Field, R., Guégan, J. F., Hawkins, B. A., ... Turner, J. R. G. (2004). Predictions and tests of climate-based hypotheses of broad-scale variation in taxonomic richness. Ecology Letters, 7(12), 1121-1134. https:/ / doi.org/10.1111/j.1461-0248.2004.00671.x FAO-Organización de las Naciones Unidas para la Alimentación y la Agricultura. (2020). Global Forest Resources Assessment 2020 - key findings. https:/ / doi.org/10.1002/2014GB005021

Ferrier, S. (2002). Mapping spatial pattern in biodiversity for regional conservation planning: Where to from here? Systematic Biology, 51(2), 331-363. 
https:/ / doi.org/10.1080/10635150252899806

Fick, S. E. \& Hijmans, R. J. (2017). WorldClim 2: new $1 \mathrm{~km}$ spatial resolution climate surfaces for global land areas. International Journal of Climatology, 37(12), 4302-4315.

Forero-Medina, G. \& Joppa, L. (2010). Representation of global and national conservation priorities by Colombia's protected area network. PLoS ONE, 5(10), 1-11. https:/ / doi.org/10.1371/journal.pone.0013210

Gardner, A. S., Maclean, I. M. D. \& Gaston, K. J. (2020). A new system to classify global climate zones based on plant physiology and using high temporal resolution climate data. Journal of Biogeography, 1-11. https://doi.org/10.1111/jbi.13927

González-Caro, S. E Vásquez, Á. (2017). Estado de los bosques de Antioquia entre 1990-2015. In E. Quintero-Vallejo, A. M. Benavides, N. Moreno, \& S. González-Caro (Eds.). Bosques Andinos, estado actual y retos para su conservación en Antioquia. (Pp. 63-80). Fundación Jardín Botánico de Medellín Joaquín Antonio Uribe.

González-Chaves, A., Jaffé, R., Metzger, J. P. \& de M. P. Kleinert, A. (2020). Forest proximity rather than local forest cover affects bee diversity and coffee pollination services. Landscape Ecology, 1. https:/ / doi.org/10.1007/s10980-020-01061-1

Haddad, N. M., Brudvig, L. A., Clobert, J., Davies, K. F., Gonzalez, A., Holt, R. D., ... Townshend, J. R. (2015). Habitat fragmentation and its lasting impact on Earth's ecosystems. Science Advances, 1(2), 1-10. https:/ / doi.org/10.1126/sciadv.1500052

Hansen, M. C., Potapov, P. V, Moore, R., Hancher, M., Turubanova, S. A. \& Tyukavina, A. (2013). High-resolution global maps of 21st-century forest cover change. Science, 342, 850-854.

Hobi, M. L., Dubinin, M., Graham, C. H., Coops, N. C., Clayton, M. K., Pidgeon, A. M. \& Radeloff, V. C. (2017). A comparison of Dynamic Habitat Indices derived from different MODIS products as predictors of avian species richness. Remote Sensing of Environment, 195, 142-152.

https:/ / doi.org/10.1016/j.rse.2017.04.018

Hobi, M. L., Farwell, L. S., Dubinin, M., Kolesov, D., Pidgeon, A. M., Coops, N. C. \& Radeloff, V. C. (2021). Patterns of bird species richness explained by annual variation in remotely sensed Dynamic Habitat Indices. Ecological Indicators, 127, 107774. https:/ / doi.org/10.1016/j.ecolind.2021.107774

Jackson, P. W. \& Kennedy, K. (2009). The global strategy for plant conservation: a challenge and opportunity for the international community. Trends in plant science, 14(11), 578-580.
Janzen, D. H. (1967). Synchronization of sexual reproduction of trees within the dry season in Central America. Evolution, 21, 620-637.

Karger, D. N., Conrad, O., Böhner, J., Kawohl, T., Kreft, H., Soria-Auza, R. W., ... Kessler, M. (2017). Climatologies at high resolution for the earth's land surface areas. Scientific Data, 4(170122), 1-20. https:/ / doi.org/10.1038/sdata.2017.122

Kattan, G. H., Franco, P., Rojas, V. \& Morales, G. (2004). Biological diversification in a complex region: $\mathrm{A}$ spatial analysis of faunistic diversity and biogeography of the Andes of Colombia. Journal of Biogeography, 31(11), 1829-1839. https:/ / doi.org/10.1111/j.1365-2699.2004.01109.x

Krebs, C. J. (1999). Ecological Methodology. An Imprint of Addison Wesley Longman (2nd ed.). Addison-Wesley Educational Publishers.

Laurance, W. F., Useche, D. C., Rendeiro, J., Kalka, M., Bradshaw, C. J. A., Sloan, S. P., ... Zamzani, F. (2012). Averting biodiversity collapse in tropical forest protected areas. Nature, 489(7415), 290-293.

https:/ / doi.org/10.1038/ nature11318

Leisher, C., Touval, J., Hess, S. M., Boucher, T. M. \& Reymondin, L. (2013). Land and forest degradation inside protected areas in Latin America. Diversity, 5(4), 779-795.

https:/ / doi.org/10.3390/d5040779

Lopes, R. H. C., Reid, I. \& Hobson, P. R. (2007). The two-dimensional Kolmogorov-Smirnov test. En XI International Workshop on Advanced Computing and Analysis Techniques in Physics Research, Nikhef12. Amsterdam, Netherlands.

Molina López, L. A. E Aristizábal Granados, J. P. (2018). Desarrollar acciones para fortalecer la conservación del hábitat y las especies de fauna silvestre en el corredor biológico del oso andino en la jurisdicción de Corantioquia (Infome técnico). eQual consultoría.

Morales, L. M., Benavides, A. M., Calderón-Caro, J. \& Zapata Giraldo, V. (2020). Pérdida de la cobertura vegetal en Antioquia (Colombia). Observatorio de Bosque de Antioquia. Recuperado de:

https:/ / observatoriobosquesantioquia.org/ficha-deforestacion-en-antioquia-2000-2019/

Morelli, T. L., Smith, A. B., Mancini, A. N., Balko, E. A., Borgerson, C., Dolch, R., ... Baden, A. L. (2020). The fate of Madagascar's rainforest habitat. Nature Climate Change, 10(1), 89-96.

https:/ / doi.org/10.1038/s41558-019-0647-x

Myers, N., Mittermeier, R. A., Mittermeier, C. G., da Fonseca, G. A. B. \& Kent, J. (2000). Biodiversity hotspots for conservation priorities. Nature, 403, 853-858. https:/ / doi.org/10.1038/35002501 
Orme, C. D. L., Davies, R. G., Burgess, M., Eigenbrod, F., Pickup, N., Olson, V. A., ... Owens, I. P. F. (2005). Global hotspots of species richness are not congruent with endemism or threat. Nature, 436(7053), 1016-1019. https:/ / doi.org/10.1038/nature03850

Piquer-Rodríguez, M., Torella, S., Gavier-Pizarro, G., Volante, J., Somma, D., Ginzburg, R. \& Kuemmerle, T. (2015). Effects of past and future land conversions on forest connectivity in the Argentine Chaco. Landscape Ecology, 30(5), 817-833. https:/ / doi.org/10.1007/s10980-014-0147-3

Politi, N., Rivera, L., Martinuzzi, S., Radeloff, V. C. \& Pidgeon, A. M. (2021). Conservation prioritization when species distribution data are scarce. Landscape and Urban Planning, 210 (August 2020), 104067. https:/ / doi.org/10.1016/j.landurbplan.2021.104067

Radeloff, V. C., Dubinin, M., Coops, N. C., Allen, A. M., Brooks, T. M., Clayton, M. K., ... Hobi, M. L. (2019). The Dynamic Habitat Indices (DHIs) from MODIS and global biodiversity. Remote Sensing of Environment, 222(February 2018), 204-214.

https:/ / doi.org/10.1016/j.rse.2018.12.009

Ramírez-Villegas, J., Cuesta, F., Devenish, C., Peralvo, M., Jarvis, A. \& Arnillas, C. A. (2014). Using species distributions models for designing conservation strategies of Tropical Andean biodiversity under climate change. Journal for Nature Conservation, 22(5), 391-404.

https:/ / doi.org/10.1016/j.jnc.2014.03.007

Ricketts, T. H., Daily, G. C., Ehrlich, P. R. \& Michener, C. D. (2004). Economic value of tropical forest to coffee production. Proceedings of the National Academy of Sciences of the United States of America, 101(34), 12579-12582.

https:/ / doi.org/10.1073/pnas.0405147101

Sandel, B., Arge, L., Dalsgaard, B., Davies, R. G., Gaston, K. J., Sutherland, W. J. \& Svenning, J.-C. (2011). The influence of late Quaternary climate-change velocity on species endemism. Science, 334, 660-664. https:/ / doi.org/10.1126/science.1210173

Slik, J. W. F., Arroyo-Rodríguez, V., Aiba, S. I., Álvarez-Loayza, P., Alves, L. F., Ashton, P., ... Venticinque, E. M. (2015). An estimate of the number of tropical tree species. Proceedings of the National Academy of Sciences of the United States of America, 112(33), E4628-E4629.

https:/ / doi.org/10.1073/ pnas.1512611112

Soria-Auza, R. W. $\mathcal{E}$ Kessler, M. (2008). The influence of sampling intensity on the perception of the spatial distribution of tropical diversity and endemism: A case study of ferns from Bolivia. Diversity and Distributions, 14(1), 123-130.

https:/ / doi.org/10.1111/j.1472-4642.2007.00433.x

Triviño, D., Silva, A., Botero, S. \& González-Caro, S. (2018). Síntesis de los registros biológicos de Antioquia a 2016. En E. Quintero-Vallejo, A. M. Benavides, N. Moreno, \& S. González-Caro (Eds.), Bosques Andinos, estado actual y retos para su conservación en Antioquia. (Pp.. 321-337). Fundación Jardín Botánico de Medellín Joaquín Antonio Uribe-Programa Bosques Andinos (COSUDE).

Venter, O., Magrach, A., Outram, N., Klein, C. J., Possingham, H. P., Di Marco, M. \& Watson, J. E. M. (2018). Bias in protected-area location and its effects on long-term aspirations of biodiversity conventions. Conservation Biology, 32(1), 127-134. https: / / doi.org/10.1111/cobi.12970

Wilson, S. J. \& Rhemtulla, J. M. (2018). Small montane cloud forest fragments are important for conserving tree diversity in the Ecuadorian Andes. Biotropica, 50(4), 586-597.

https:/ / doi.org/10.1111/btp.12542

Wright, D. H. (1983). Species-energy theory : an extension of species-area theory. Oikos, 41(3), 496-506.

Young, I. T. (1977). Proof without prejudice: use of the Kolmogorov Smirnov test for the analysis of histograms from flow systems and other sources. Journal of Histochemistry and Cytochemistry, 25(7), 935-941. https:/ / doi.org/10.1177/25.7.894009

Zhai, D. L., Cannon, C. H., Dai, Z. C., Zhang, C. P. \& Xu, J. C. (2015). Deforestation and fragmentation of natural forests in the upper Changhua watershed, Hainan, China: implications for biodiversity conservation. Environmental Monitoring and Assessment, 187(4137), 2-12.

https:/ / doi.org/10.1007/s10661-014-4137-3 


\section{Material suplementario}

Tabla S1. Descripción de las AP dentro del Corredor del Oso de Anteojos (COA), en Antioquia, Colombia. En negrilla están resaltadas las AP con mayor extensión, cobertura de bosque y deforestación. Los usos sostenible y público hacen referencia al desarrollo de actividades agrícolas, ganaderas, mineras, forestales, industriales, y proyectos de desarrollo de manera controlada y sostenible, así como actividades de recreación y ecoturismo (Decreto 2372 de 2010). DRMI: Distrito Regional de Manejo Integrado. PNN: Parque Nacional Natural. PNR: Parques Naturales Regionales. RFPN: Reserva Forestal Protectora Nacional. RNSC: Reserva Natural de la Sociedad Civil.

Table S1. Description of the protected areas within the Spectacled Bear Corridor (COA) protected areas in Antioquia, Colombia. Protected areas with the largest extension, forest cover and deforestation are in bold. Sustainable and public uses refer to the development of agricultural, livestock, mining, forestry, industrial activities, and development projects in a regulated and sustainable way, as well as recreation and ecotourism activities (Decree 2372 of 2010). DRMI: Regional Integrated Management District. NNP: National Natural Park. PNR: Regional Natural Parks. RFPN: National Protected Forest Reserve. RNSC: Civil Society Natural Reserve.

\begin{tabular}{|c|c|c|c|c|c|c|c|}
\hline Áreas protegidas & $\begin{array}{l}\text { Área } \\
(\mathbf{k m} 2)\end{array}$ & $\begin{array}{l}\text { Precipita- } \\
\text { ción anual } \\
\text { ( } \mathrm{mm} \text { año-1) }\end{array}$ & $\begin{array}{l}\text { Tempera- } \\
\text { tura media } \\
\text { anual }\left({ }^{\circ} \mathrm{C}\right)\end{array}$ & $\begin{array}{l}\text { Eleva- } \\
\text { ción } \\
(\mathrm{msnm})\end{array}$ & $\begin{array}{c}\text { Bosque } \\
(\mathbf{k m} 2)\end{array}$ & $\begin{array}{l}\text { Deforesta- } \\
\text { ción }(\mathbf{k m} 2)\end{array}$ & $\begin{array}{c}\text { Usos } \\
\text { permitidos }\end{array}$ \\
\hline DRMI Alto del Insor & 69.00 & 2086.61 & 12.86 & 2572.60 & 55.91 & 0.906 & \multirow{3}{*}{$\begin{array}{l}\text { Preservación, } \\
\text { restauración, } \\
\text { sostenible y } \\
\text { público }\end{array}$} \\
\hline $\begin{array}{l}\text { DRMI Cuchilla Cerro } \\
\text { Plateado Alto San José }\end{array}$ & 88.44 & 3036.71 & 14.50 & 2306.85 & 50.48 & 3.70 & \\
\hline $\begin{array}{l}\text { DRMI Cuchilla Jardín } \\
\text { Támesis }\end{array}$ & 271.49 & 2580.10 & 14.23 & 2454.44 & 156.83 & 3.71 & \\
\hline PNN Las Orquídeas & 287.49 & 2413.64 & 17.47 & 1714.13 & 265.85 & 3.00 & \multirow{5}{*}{$\begin{array}{l}\text { Preservación } \\
\text { y } \\
\text { restauración } \\
\text { Preservación, } \\
\text { Restauración, } \\
\text { sostenible y } \\
\text { público }\end{array}$} \\
\hline $\begin{array}{l}\text { PNR Corredor de las } \\
\text { Alegrías }\end{array}$ & 100.81 & 2154.30 & 11.85 & 2811.62 & 80.91 & 1.18 & \\
\hline RFPN Carauta & 267.06 & 2558.01 & 20.28 & 1188.24 & 226.54 & 8.45 & \\
\hline RFPN De Urrao & 284.06 & 2147.51 & 13.20 & 2480.21 & 213.73 & 4.64 & \\
\hline $\begin{array}{l}\text { RFPN Farallones del } \\
\text { Citará }\end{array}$ & 291.44 & 3127.09 & 13.55 & 2535.73 & 209.06 & 12.27 & \\
\hline RNSC Buena Vista & 0.09 & 2916.50 & 15.30 & 2036.22 & 0.02 & 0.017 & \\
\hline RNSC Colibrí del Sol & 1.65 & 2263.00 & 8.97 & 3355.20 & 0.58 & 0.001 & \\
\hline $\begin{array}{l}\text { RNSC de las Aves Coli- } \\
\text { brí del Sol }\end{array}$ & 13.30 & 2116.38 & 9.13 & 3267.05 & 3.72 & 0.041 & \\
\hline RNSC La Violeta & 0.0035 & 2114.00 & 16.50 & 1911.00 & 0.003 & - & \\
\hline RNSC Orquídeas & 1.74 & 2601.67 & 12.13 & 2921.71 & 1.61 & - & \\
\hline
\end{tabular}


Figura S1. Mapas de localización, cobertura de bosque, deforestación, grupos climáticos y fragmentación en el Corredor del Oso de Anteojos (COA), en Antioquia, Colombia. a, presencia y ausencia de bosque, y deforestación, con una resolución espacial de $30 \mathrm{~m} \times 30 \mathrm{~m}$; b, grupos climáticos y sus áreas de importancia biológica (IB); c, fragmentación para una ventana de análisis de $3 \mathrm{~km} \times 3 \mathrm{~km}$ y los remanentes de bosque sin fragmentación dentro del COA. AP, Áreas protegidas; IB, Importancia biológica. Sistema de coordenadas: MAGNA Colombia Bogotá

Figure S1. Maps of location, forest cover, deforestation, climate groups and fragmentation of the Spectacled Bear Corridor (COA) protected areas in Antioquia, Colombia. a, presence and absence of forest, and deforestation with a spatial resolution of $30 \mathrm{~m} \times 30 \mathrm{~m}$; b, climate groups and their biological importance areas; $c$, fragmentation for a $3 \mathrm{~km} \times 3 \mathrm{~km}$ analysis window, and the remaining forest without fragmentation within the COA. AP, protected areas; IB, Biological importance. Coordinate system: MAGNA Colombia Bogotá.
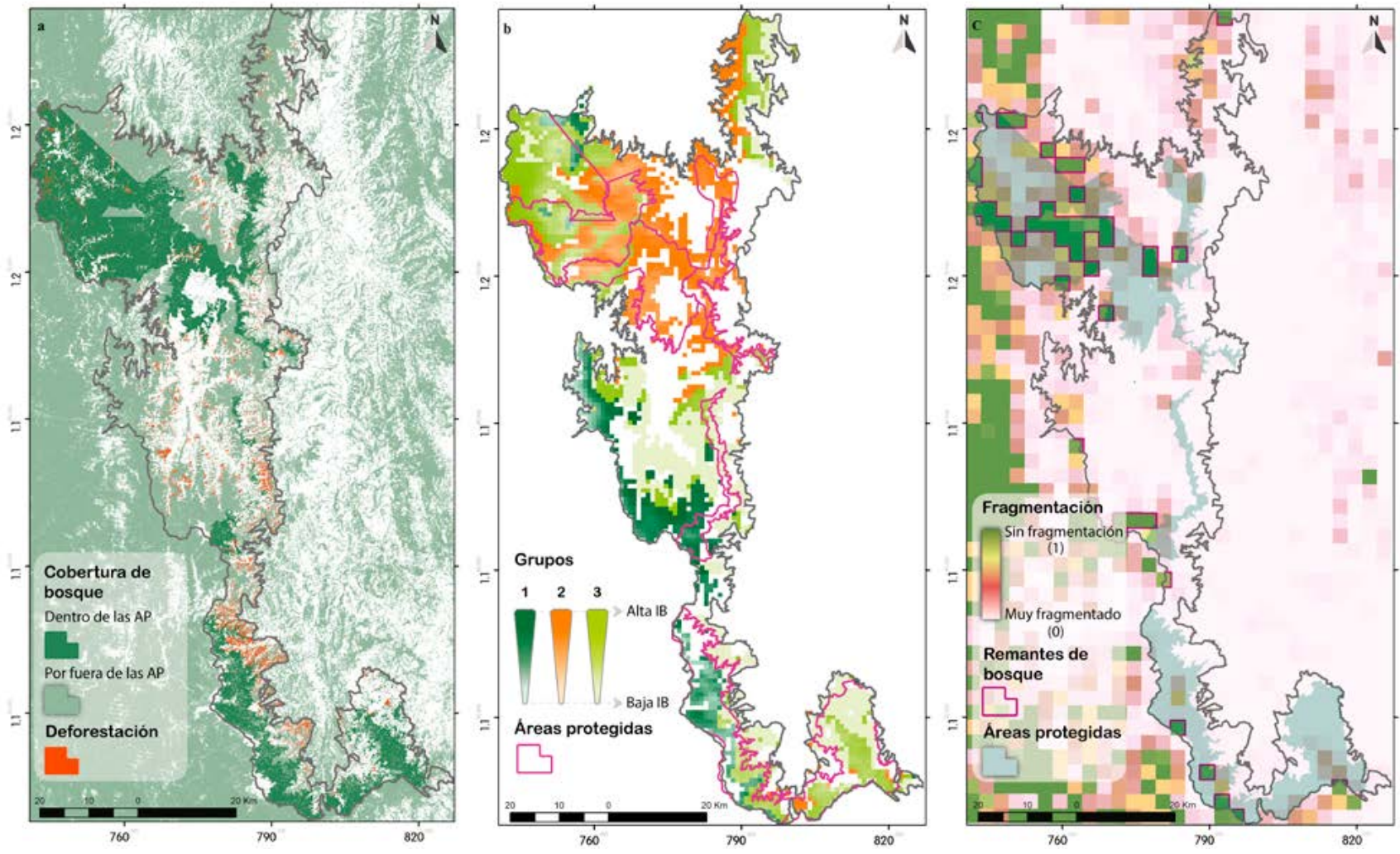
Jennifer Calderón-Caro

Fundación Jardín Botánico Joaquín Antonio Uribe de Medellín. Medellín. Colombia.

jennifer.calderon@jbotanico.org

https:/ / orcid.org/0000-0001-9222-1203

\section{Ana María Benavides}

Fundación Jardín Botánico Joaquín Antonio Uribe de Medellín. Medellín. Colombia.

https:/ / orcid.org/0000-0002-1189-9379
Deforestación y fragmentación en las áreas más biodiversas de la Cordillera Occidental de Antioquia (Colombia)

Citación: Calderón-Caro, J. y Benavides, A.M. (2022). Deforestación y fragmentación en las áreas más biodiversas de la Cordillera Occidental de Antioquia (Colombia). Biota Colombiana, 23(1), e942.

\section{https://doi.org/10.21068/2539200X.942}

Recibido: 20 de enero 2021

Aceptado: 17 de septiembre 2021 